OPEN ACCESS

Edited by:

J. Luis Espinoza,

Kindai University, Japan

Reviewed by:

Wen Zhou,

Case Western Reserve University,

United States

Greg Woods,

University of Tasmania, Australia

*Correspondence:

Hsuan-Liang Liu

f10894@ntut.edu.tw

Shwu-Jiuan Lin

shwu-lin@tmu.edu.tw

Specialty section:

This article was submitted to Pharmacology of Anti-Cancer Drugs,

a section of the journal

Frontiers in Pharmacology

Received: 05 March 2018

Accepted: 04 July 2018

Published: 02 August 2018

Citation:

Ho Y, Chen Y-F, Wang L-H, Hsu K-Y,

Chin $Y-T$, Yang $Y-C S H$, Wang $S-H$,

Chen Y-R, Shih Y-J, Liu LF, Wang K,

Whang-Peng J, Tang $\mathrm{H}-\mathrm{Y}$, Lin $\mathrm{H}-\mathrm{Y}$,

Liu H-L and Lin S-J (2018) Inhibitory

Effect of Anoectochilus formosanus

Extract on Hyperglycemia-Related

PD-L1 Expression and Cancer

Proliferation. Front. Pharmacol. 9:807.

doi: 10.3389/fphar.2018.00807

\section{Inhibitory Effect of Anoectochilus formosanus Extract on Hyperglycemia-Related PD-L1 Expression and Cancer Proliferation}

\author{
Yih Ho', Yan-Fang Chen', Li-Hsuan Wang 1,2, Kuang-Yang Hsu', Yu-Tang Chin ${ }^{3,4}$, \\ Yu-Chen S. H. Yang ${ }^{5}$, Shwu-Huey Wang ${ }^{6}$, Yi-Ru Chen ${ }^{3,4}$, Ya-Jung Shih ${ }^{3,4}$, Leroy F. Liu ${ }^{3}$, \\ Kuan Wang ${ }^{7}$, Jacqueline Whang-Peng ${ }^{3}$, Heng-Yuan Tang ${ }^{8}$, Hung-Yun Lin ${ }^{3,4,8,9}$, \\ Hsuan-Liang Liu' ${ }^{10 *}$ and Shwu-Jiuan Lin ${ }^{1 *}$
}

${ }^{1}$ School of Pharmacy, Taipei Medical University, Taipei, Taiwan, ${ }^{2}$ Department of Pharmacy, Taipei Medical University Hospital, Taipei, Taiwan, ${ }^{3}$ Taipei Cancer Center, College of Medical Science and Technology, Taipei Medical University, Taipei, Taiwan, ${ }^{4}$ PhD Program for Cancer Molecular Biology and Drug Discovery, College of Medical Science and Technology, Taipei Medical University, Taipei, Taiwan, ${ }^{5}$ Joint Biobank, Office of Human Research, Taipei Medical University, Taipei, Taiwan, ${ }^{6}$ Core Facility Center, Office of Research and Development, Department of Biochemistry and Molecular Cell Biology, College of Medicine, Taipei Medical University, Taipei, Taiwan, ${ }^{7}$ Graduate Institute of Nanomedicine and Medical Engineering, College of Medical Engineering, Taipei Medical University, Taipei, Taiwan, ${ }^{8}$ Pharmaceutical Research Institute, Albany College of Pharmacy and Health Sciences, Albany, NY, United States, ${ }^{9}$ TMU Research Center of Cancer Translational Medicine, Taipei Medical University, Taipei, Taiwan, ${ }^{10}$ Department of Chemical Engineering and Biotechnology, Institute of Biochemical and Biomedical Engineering, National Taipei University of Technology, Taipei, Taiwan

Traditional herb medicine, golden thread (Anoectochilus formosanus Hayata) has been used to treat various diseases. Hyperglycemia induces generation of reactive oxygen species (ROS) and enhancement of oxidative stress which are risk factors for cancer progression and metastasis. In this study, we evaluated hypoglycemic effect of $A$. formosanus extracts (AFEs) in an inducible hyperglycemia animal model and its capacity of free-radical scavenging to establish hyperglycemia-related carcinogenesis. AFE reduced blood glucose in hyperglycemic mice while there was no change in control group. The incremental area under blood glucose response curve was decreased significantly in hyperglycemic mice treated with AFE in a dose-dependent manner. AFE and metformin at the same administrated dose of $50 \mathrm{mg} / \mathrm{kg}$ showed similar effect on intraperitoneal glucose tolerance test in hyperglycemic mice. Free-radical scavenger capacity of AFE was concentration dependent and $200 \mu \mathrm{g} / \mathrm{ml}$ of AFE was able to reduce more than $41 \%$ of the free radical. Treatment of cancer cells with AFE inhibited constitutive $P D-L 1$ expression and its protein accumulation. It also induced expression of pro-apoptotic genes but inhibited proliferative and metastatic genes. In addition, it induced anti-proliferation in cancer cells. The results suggested that AFE not only reduced blood glucose concentration as metformin but also showed its potential use in cancer immune chemoprevention/therapy via hypoglycemic effect, ROS scavenging and $P D-L 1$ suppression.

Keywords: hyperglycemia, metformin, $P D-L 1$, anti-proliferation, cancer 


\section{INTRODUCTION}

Hyperglycemia is the most prominent sign that characterizes diabetes (Kitabchi et al., 2009). Reduced liver, fat, and muscle uptake of glucose and resultant hyperglycemia are caused by reduced insulin production or cellular resistance to insulin action or a combination of these factors. Evidence confirms that inflammation plays a key role in pathogenesis of diabetes mellitus (Navarro and Mora, 2005; Agrawal and Kant, 2014). Targeting the inflammation and its signaling pathways may be effective to manage diabetes mellitus and its associated complications including nephropathy, ischemic heart disease, peripheral vascular disease, and cerebrovascular disease (Gothai et al., 2016). Results of numerous epidemiological studies indicate that diabetic population is not only at increased risk of cardiovascular complications but also at substantially higher risk of many forms of malignancies (Kasznicki et al., 2014). However, the underlying mechanisms are poorly understood.

A long-term hyperglycemia is likely to lead to many complexation accompany syndromes, such as blindness, renal failure, vascular disease (Cade, 2008), and other diseases such as cancers (Duan et al., 2014; Ryu et al., 2014). Therefore, blood glucose concentrations must be maintained within a certain range, especially during those of fasting and $2 \mathrm{~h}$ after meal. In addition, hyperglycemia could also modulate expression of epithelial-mesenchymal transition (EMT)-related factors in pancreatic tumor tissues, as E-cadherin level is decreased and the expression of mesenchymal markers $\mathrm{N}$-cadherin and vimentin, as well as transcription factor snail, are strongly increased (Li et al., 2016). Fructose is easy to restore linear. The Maillard reaction of reducing sugars' carbonyls and proteins into Henry's product can catalyze the conversion of sugar to a superoxide free radical and cause cell damage. In vitro tests found that fructose saccharifications faster than glucose, indeed seven times faster, so fructose production of reactive oxygen species (ROS) is also much higher than that of glucose. ROS, including hydrogen peroxide, generated by mitochondrial respiratory chain is a number of chemically reactive molecules derived from oxygen, which play a significant role in the initiation and progression of cancer (Trachootham et al., 2009). Intracellular ROS can motivate tumor progression by promoting cell proliferation, survival, invasion, and metastasis (Nishikawa et al., 2009). In addition to hyperglycemic condition, superoxide dismutase (SOD) is able to induce mild ROS production to promote invasive and migratory activities of pancreatic cancer ( $\mathrm{Li}$ et al., 2011, 2015). ERK1/2 signaling pathways are important signaling cascades downstream of ROS that is involved in tumor migration and invasion ( $\mathrm{Wu}$ et al., 2008).

Metformin, the most commonly prescribed drug for type II diabetes, is constantly associated with decreased risk of the occurrence of various types of cancers, especially of pancreas (Rahn et al., 2018), colon (Anisimov, 2016), and hepatocellular carcinoma (Donadon et al., 2010). This observation is also confirmed by the results of numerous metaanalyses. Hyperglycemia has been shown to induce changes in neutrophil mobilization, primary tumor growth, and metastatic progression (Fainsod-Levi et al., 2017). It is also associated with significantly higher rates of cancer-related deaths, particularly in gastrointestinal cancer and leukemia, but not with non-cancerrelated deaths (Simon et al., 2018).

The checkpoint programmed death-1/PD-ligand 1 (PD-1/PDL1) is a spirited regulator for the interaction of activated $T$ cell and tumor cells. Overexpression of PD-L1 has been shown to protect tumor cells against immune demolition. It engages PD-1 to reverse activated $\mathrm{T}$ cell interaction with tumor cells and may also serve to induce apoptosis of T cells. Overexpression of PD-L1 is observed in melanoma (Thierauf et al., 2015), pancreatic cancer (Zhuan-Sun et al., 2017), oral cancer (Goncalves et al., 2017), and colorectal cancer (Droeser et al., 2013; Thierauf et al., 2015). It can be induced by thyroid hormone and other growth factors. We have shown that thyroid hormone analog, tetrac can block expression of PD-L1 (Lin et al., 2016). In addition, anti-oxidants such as resveratrol (Chin et al., 2018) and curcumin (Lim et al., 2016) have been reported to inhibit PD-L1 expression.

Anoectochilus formosanus Hayata (Orchidaceae) is a native folk medicine in Taiwan. It is a perennial herb which whole plant can be used as a medicine. It was also used to treat bruises and poisonous snake bites. Currently, it also used as dehumidification and detoxification and had effects in treating hyperactivity cough, tuberculosis vomiting blood, hematuria, hypertension, pediatric shock, tetanus, and nephritis edema. Studies have pointed out that water extract of $A$. formosanus contains many antioxidant components (Shih et al., 2003), which reduce lipid peroxides-induced hyperglycemia and reduce streptozotocin-induced diabetes in rats with fasting blood glucose (Shih et al., 2002). However, there is no report on the hypoglycemic effects of $A$. formosanus on fructose-induced hyperglycemic mice. In the present study, the hypothesis is that $A$. formosanus extract (AFE) may be able to inhibit hyperglycemia-induced production of ROS, to reduce blood glucose and to suppress the expression of $P D-L 1$ in fructoseinduced hyperglycemic mice model for the cancer prevention. Interestingly, results indicate that AFE has the same effect as metformin in reducing blood sugar. AFE inhibited PD-L1 expression and its protein accumulation. In addition, it inhibited expression of proliferative genes such as cell proliferation in oral cancer cells. These results suggest that AFE can be used in cancer immune chemoprevention or chemotherapy in the future.

\section{MATERIALS AND METHODS}

\section{Preparation of AFE}

The whole plants of $A$. formosanus Hayata were collected from the mountain area of Puli Township, Nantou County, Taiwan and were authenticated by Prof. Kur-Ta Cheng, Department of Biochemistry, Taipei Medical University. The fresh whole plants were frozen overnight and then oven-dried under $60^{\circ} \mathrm{C}$. The dried whole plants were extracted with methanol at room temperature and filtered. The filtrate was then evaporated under reduced pressure to give the AFE. 


\section{Cell Culture}

Human oral cancer SCC-25 cells were obtained from American Type Culture Collection (ATCC) (Manassas, VA, United States). The cell line had been tested (isoenzyme analysis, mycoplasma, cytogenetics, tumorigenesis, and receptor expression testing) and authenticated with BCRC (The Bioresource Collection and Research Center, Taiwan). Cells were maintained in RPMI1640 supplemented with $10 \%$ FBS. Incubation conditions were $5 \% \mathrm{CO}_{2}$ at $37^{\circ} \mathrm{C}$. Before the study, cells were placed in $0.25 \%$ hormone-depleted serum-supplemented medium for 2 days.

\section{Animal}

The animal protocol was approved by the Laboratory Animal Care and Use Committee of Taipei Medical University, Animal Experiment Application No. LAC-100-0074. C57BL/6 mice aged 4 weeks were purchased from National Laboratory Animal Center of National Taiwan University and were housed and experimented at Animal Research Center of Taipei Medical University. The temperature of animal breeding room was controlled at $22^{\circ} \mathrm{C}$ and illumination time was $12 \mathrm{~h}$ (7:00-19:00), give adequate amounts of reverse osmosis water and lab diet feed (crude protein more than $20 \%$, crude fat more than $9 \%$, and crude fiber less than $4 \%$ ) feeding. All the fasting blood sampling and intraperitoneal glucose tolerance test (IGTT) experiments were performed during 19:30 to 22:00 to fit circadian rhythm of mice.

\section{Hyperglycemia-Induced in Mice}

Thirty mice were randomly divided into six cages. There was no experiment in first 2 weeks to allow mice adapt to the environment. The mice were then divided into two groups control group $(n=10)$ and experimental group. Drinking water was given to control group while fructose containing water was given to the experimental group. The fructose drinking water for experimental group was $5 \%$ on first day, and progressively increased to $25 \%$ on every second day. The drinking water was changed every 2 days. The feeding process continuously records mouse drinking and dietary conditions. The fasting blood glucose concentrations were examined in each group beginning from 4 th-week induction until the significant difference in blood glucose concentration of two groups was obtained.

\section{Intraperitoneal Glucose Tolerance Test}

The mice in both control and experimental group were fasting for $12 \mathrm{~h} \mathrm{(7:00} \mathrm{to} \mathrm{19:00)} \mathrm{and} \mathrm{tail} \mathrm{vein} \mathrm{blood} \mathrm{was} \mathrm{collected} \mathrm{as} \mathrm{fasting}$ glucose baseline before each IGTT experiment. Three doses (10, 25 , and $50 \mathrm{mg} / \mathrm{kg}$ body weight) dosages of AFE prepared in normal saline with Tween 20 were injected intraperitoneally before glucose (1 $\mathrm{g} / \mathrm{kg}$ body weight) was given. The blood glucose levels were measured at 0, 30, 60, and 120 min after glucose was given. The changes of blood glucose level and the incremental areas under the blood glucose curves ( $\triangle \mathrm{AUCs}$ ) were calculated.

\section{Free Radical-Scavenging Assay}

The 5,5-dimethyl-1-pyrroline- $N$-oxide (DMPO), a common spin trap, was used to react with $\mathrm{O}_{-}, \mathrm{N}$-, $\mathrm{S}$-, and $\mathrm{C}$-centered radicals to form stable adducts that can be detected by electron spin resonance (ESR, EMX-6/1 ESR Spectrometer, Bruker, Karlsruhe, Germany) to study capacity of free radical scavenging ability of AFE. Ferrous was reacted with hydrogen peroxide to oxidize into ferric and formed a hydroxyl radical (HO•) and a hydroxide ion in the process. Then ferric was reduced back to ferrous by AFE through another molecule of hydrogen peroxide to form a hydroperoxyl radical (HOO•). Then, free radicals generated by this process then were detected by ESR to obtain typical 1:2:2:1 intensity ratio of $\mathrm{DMPO} / \bullet \mathrm{OH}$ four line spectrum. The intensity of the second line was taken to calculate relative intensities of various AFE sample to DMPO.

\section{Quantitative Real-Time PCR}

Oral cancer SCC-25 cells were set in Petri dishes and starved for $48 \mathrm{~h}$ before being treated with AFE and metformin. SCC-25 cells were treated with refreshed media with agents daily for 3 days. Total RNA was extracted and genomic DNA was also eliminated with Illustra RNAspin Mini RNA Isolation Kit (GE Healthcare Life Sciences, Buckinghamshire, United Kingdom). One microgram of DNase I-treated total RNA was reversetranscribed with RevertAid $\mathrm{H}$ Minus First Strand cDNA Synthesis Kit (Life Technologies Corp.) into cDNA and used as the template for real-time PCR reactions and analysis. The real-time PCR reactions were performed using QuantiNovaTM SYBR $^{\circledR}$ Green PCR Kit (QIAGEN, Hilden, Germany) on CFX Connect ${ }^{\mathrm{TM}}$ Real-Time PCR Detection System (Bio-Rad Laboratories, Inc., Hercules, CA, United States). This involved an initial denaturation at $95^{\circ} \mathrm{C}$ for $5 \mathrm{~min}$, followed by 40 cycles of denaturing at $95^{\circ} \mathrm{C}$ for $5 \mathrm{~s}$ and combined annealing/extension at $60^{\circ} \mathrm{C}$ for $10 \mathrm{~s}$, as detailed in the manufacturer's instructions. The primer sequences were shown as the following: Homo sapiens PD-L1, forward 5'-GTTGAAGGACCAGCTCTCCC- $3^{\prime}$ and reverse 5'-ACCCCTGCATCCTGCAATTT-3' (Accession No. AY254342.1); Homo sapiens cyclooxygenase 2 (COX-2), forward 5'-GCCAAGCACTTTTGGTGGAG-3' and reverse 5'-GGGACA GCCCTTCACGTTAT-3' (Accession No. AY462100.1); Homo sapiens tumor necrosis factor-alpha (TNF- $\alpha)$, forward: 5'-CCTGCTGCACTTTGGAGTGA-3', reverse: 5' -TCGAGA AGATGATCTGACTGCC-3' (Accession No. NM_000594.3); Homo sapiens cyclin D1 (CCND1) forward 5'-CAAGGCCTGAA CCTGAGGAG-3' and reverse 5'-GATCACTCTGGAGA GGAAGCG-3' (Accession No. NM_053056.2); Homo sapiens $\mathrm{v}$-myc avian myelocytomatosis viral oncogene homolog $(c-M y c)$, forward 5'-TTCGGGTAGTGGAAAACCAG-3' and reverse 5'-CAGCAGCTCGAATTTCTTCC-3' (Accession No. NM_002467); Homo sapiens matrix metalloproteinase 2 $(M M P-2)$, forward $5^{\prime}$-ATCCAGACTTCCTCAGGCGG-3' and reverse 5' -CCTGGCAATCCCTTTGTATGTT-3' (Accession No. NM_004530.5); Homo sapiens BCL2-associated agonist of cell death (BAD), forward 5' - CTTTAAGAAGGGACTTCCTCGCC$3^{\prime}$ and reverse 5'-AAGTTCCGATCCCACCAGGA-3' (Accession No. NM_032989.2); human caspase 2, apoptosis-related cysteine 
peptidase (CASP2), forward 5' -GCATGTACTCCCACCGTTGA$3^{\prime}$ and reverse $5^{\prime}$-GACAGGCGGAGCTTCTTGTA-3' (Accession No. NM_032982.3); Homo sapiens 18S rRNA gene (18S), forward 5'-GTAACCCGTTGAACCCCATT-3' and reverse 5'-CCATCCAATCGGTAGTAGCG-3' (Accession No. M10098). Calculations of relative gene expression (normalized to $18 \mathrm{~S}$ reference gene) were performed according to the $\Delta \mathrm{CT}$ method. Fidelity of the PCR reaction was determined with melting temperature analysis.

\section{Western Blotting}

To examine the effects of AFE or metformin on the expression of PD-L1, Western blot analysis was conducted to quantify protein expression levels of PD-L1 in SCC-25 cells. For Western blot analyses, cells were lysed and extracted-protein samples were separated on $10 \%$ sodium dodecyl sulfate-polyacrylamide gel (SDS-PAGE). A $60 \mu \mathrm{g}$ quantity of protein was loaded in each well with $5 \mathrm{X}$ sample buffer and the samples were resolved with electrophoresis at $100 \mathrm{~V}$ for $2 \mathrm{~h}$. The resolved proteins were transferred from the polyacrylamide gel to Millipore ImmobilonPSQ Transfer nitrocellulose membranes (Millipore, Billerica, MA, United States) with the Trans-Blot ${ }^{\circledR}$ SD Semi-Dry Transfer Cell (Bio-Rad Laboratories, Inc.). Membranes were blocked with a solution of $2 \%$ bovine serum albumin (BSA) in Tris-buffered saline. Membranes were incubated with first antibodies to PDL1 (Cell Signaling Technology, Inc., Beverly, MA, United States) and GAPDH (GeneTex International Corp., Hsinchu City, Taiwan) overnight at $4^{\circ} \mathrm{C}$. The proteins were detected with HRPconjugated secondary antibodies and Immobilon ${ }^{\mathrm{TM}}$ Western HRP Substrate Luminol Reagent (Millipore). Western blots were visualized and recorded with the Amersham Imager 600 (GE Healthcare Life Sciences, Pittsburgh, PA, United States). The densitometric analysis of Western blot was conducted by Image 1.47 software (National Institute of Health, United States) according to the software instruction.

\section{Cell Viability Assay}

SCC-25 cells were plated at a density of $10^{4}$ cells/well in 96-well plates. Cell viability was determined by using the CyQUANT ${ }^{\circledR}$ NF Cell Proliferation Assay Kit (Molecular Probes, Eugene, OR, United States) at $96 \mathrm{~h}$ after treatment. AFE or metformin was replaced daily with refreshed media. Briefly, medium was removed, and cells were incubated with CyQUANT ${ }^{\circledR}$ NF reagent for $1 \mathrm{~h}$ at $37^{\circ} \mathrm{C}$ according to the manufacturer's instructions. Plates were then analyzed by using a microplate reader (Varioskan $^{\mathrm{TM}}$ Flash Multimode Reader, Thermo Scientific, Waltham, MA, United States) (excitation, $485 \mathrm{~nm}$; emission, $530 \mathrm{~nm})$.

\section{Statistical Analysis}

Studies were analyzed with IBM ${ }^{\circledR}$ SPSS ${ }^{\circledR}$ statistics software (SPSS, Chicago, IL, United States). One-way analysis of variance was conducted for multiple group comparison and $p$-value $<0.05$ was considered statistically significant.

\section{RESULTS}

\section{Anoectochilus formosanus Extract Reduces Fructose-Induced Hyperglycemia in Mice}

In order to examine reducing effect of AFE on hyperglycemia in fructose-induced hyperglycemic mice, mice were given fructose water to induce hyperglycemia. The mice were assigned randomly to control and experimental groups at 6 weeks of age with fasting blood glucose values of $96.2 \pm 18.8$ and $102.4 \pm 22.1 \mathrm{mg} / \mathrm{dl}$, respectively. Blood glucose values were $133.0 \pm 6.0$ and $175.6 \pm 20.4 \mathrm{mg} / \mathrm{dl}$ after fed with water or fructose-containing water for 11 weeks, respectively. The significant mean difference of blood glucose value was $42.6 \mathrm{mg} / \mathrm{dl}$ between control and fructose-induced hyperglycemic mice $(p<0.001)$ after 11 weeks induction. Fasting blood glucose tests were conducted during the experimental period and blood

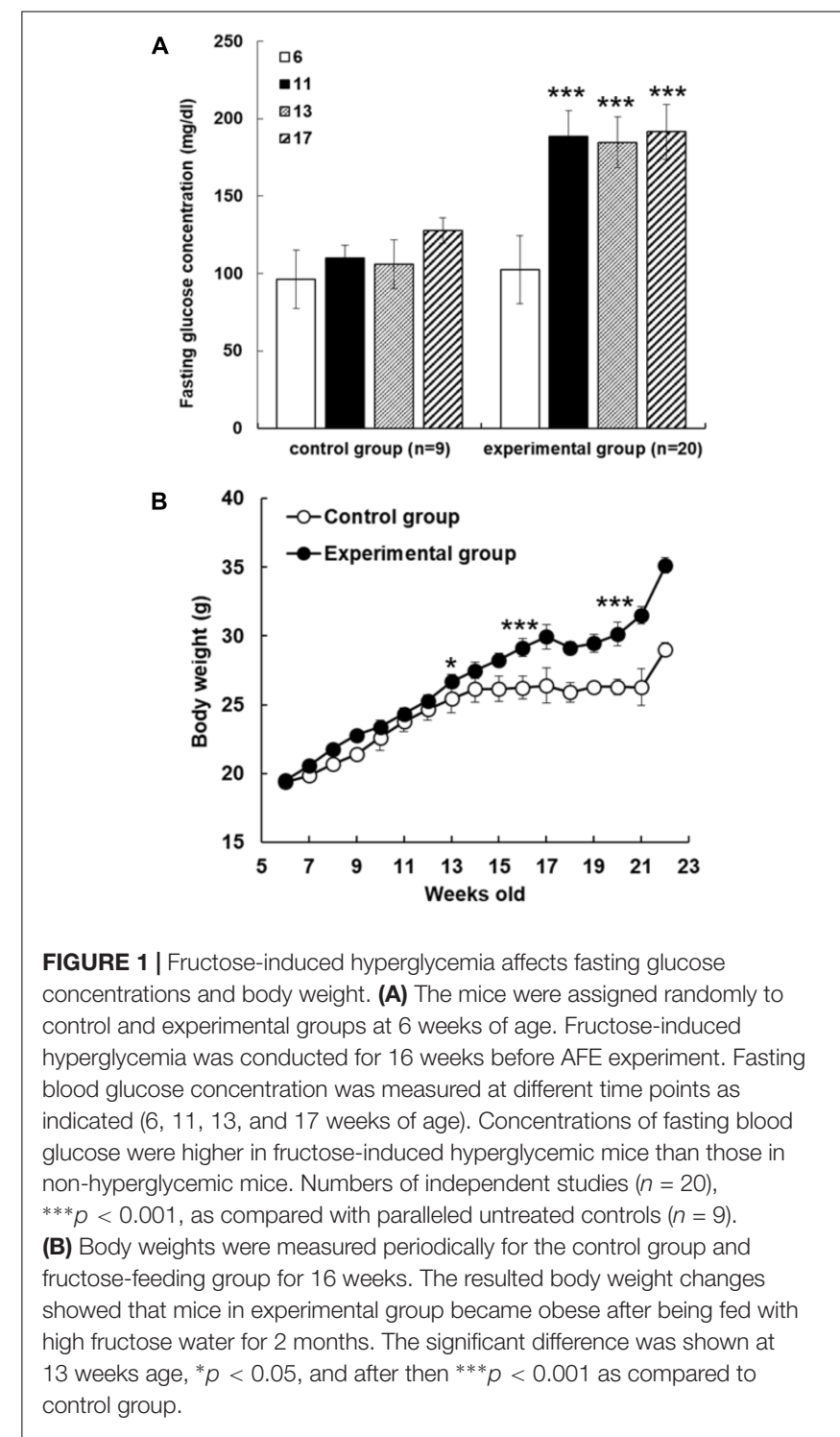



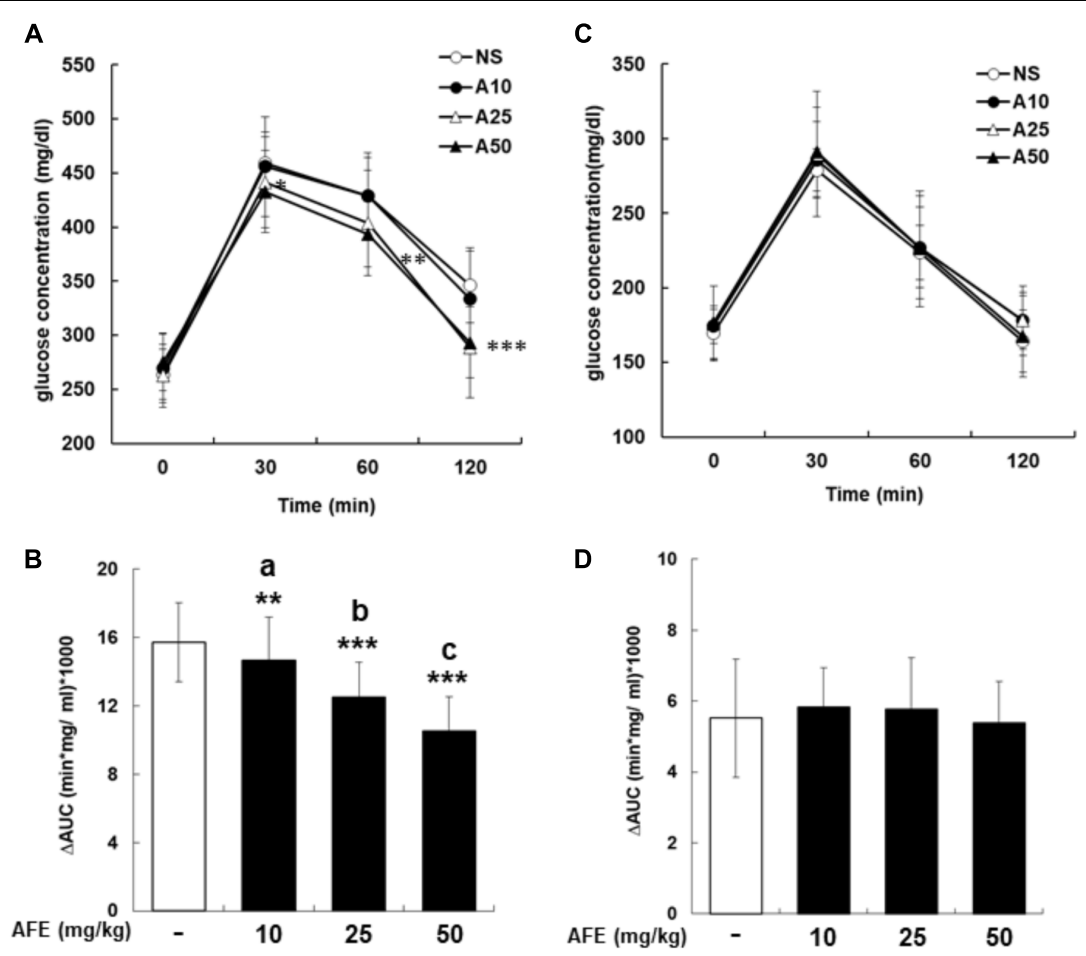

FIGURE 2 | Anoectochilus formosanus extract reduces blood glucose in hyperglycemic but not in control mice. Hyperglycemic mice were injected with different dosages of AFE intraperitoneally. Glucose was injected intraperitoneally immediately after AFE administration. Blood glucose was measured at 0, 30, 60, and 120 min after glucose administration. (A) The time courses of blood glucose concentration measured in fructose-induced hyperglycemic mice treated with different dosages of AFE (10 to 50 mg/kg). NS: treated with normal saline; A10: treated with AFE 10 mg/kg; A25: treated with AFE 25 mg/kg; A50: treated with AFE $50 \mathrm{mg} / \mathrm{kg}$. Data are shown as mean $\pm \mathrm{SD}(n=16)$. (B) Measurement of the area under the incremental blood glucose concentration curve in the glucose tolerance test of the experimental group mice. -: treated with normal saline as control; 10: treated with AFE 10 mg/kg; 25: treated with AFE 25 mg/kg; 50 : treated with AFE $50 \mathrm{mg} / \mathrm{kg}$. Data are shown as mean $\pm \mathrm{SD}$ of each group of four animals random cross over four times, analysis by one-way ANOVA test. $* * p<0.01,{ }^{* * *} p<0.001$ as compared with untreated control. ${ }^{\mathrm{a}} \mathrm{AFF} 10 \mathrm{vs} .50 \mathrm{mg} / \mathrm{kg}, p<0.001 ;{ }^{\mathrm{b}} \mathrm{AFF} 25 \mathrm{vs} .10 \mathrm{mg} / \mathrm{kg}, p<0.05 ;{ }^{\mathrm{c} A F F} 50 \mathrm{vs} .25 \mathrm{mg} / \mathrm{kg}, p<0.05$. Glucose was injected intraperitoneally after AFE administration in non-hyperglycemic mice. Blood glucose was measured at 0, 30, 60, and 120 min after glucose administration. (C) Time courses of blood glucose concentration measured in non-hyperglycemic mice treated with different dosages of AFE (10 to $50 \mathrm{mg} / \mathrm{kg}$ ). NS: normal saline; A10: treated with AFE 10 mg/kg; A25: treated with AFE 25 mg/kg; A50: treated with AFE 50 mg/kg. Data are shown as mean \pm SD ( $n=8$ ). (D) Measurement of the area under the incremental blood glucose concentration curve in the glucose tolerance test of non-hyperglycemic mice. -: treated with normal saline as control; 10 : treated with AFE $10 \mathrm{mg} / \mathrm{kg}$; 25: treated with AFE 25 mg/kg; 50: treated with AFE $50 \mathrm{mg} / \mathrm{kg}$. Data are shown as mean \pm SD, analysis by one-way ANOVA test ( $n=8$ ).

glucose gradually increased in both control and experimental group. However, fasting blood glucose was up sharply the value in fructose water feeding mice (Figure 1A). After 11 weeks of experimental induction, there was a great difference in fasting blood glucose between the two groups (Figure 1A), and the resulted body weight changes showed that mice in experimental group became obese after being fed with high fructose water for 2 months (Figure 1B).

To investigate effect of AFE on glucose tolerance test in hyperglycemic mice, mice were started glucose tolerance test at the $23 \mathrm{rd}$ week for four consecutive weeks. The experiment was designed as a crossover test. After fasting blood sample being taken, AFE was intraperitoneally administered as single dosage at 10,25 , and $50 \mathrm{mg} / \mathrm{kg}$ body weight of mice to observe the changes in blood glucose of mice within $2 \mathrm{~h}$. Glucose solution (1 $\mathrm{g} / \mathrm{kg}$ ) was given immediately after AFE injected to mice. Blood samples were collected from tail vein for blood glucose measurement at 30,60, and $120 \mathrm{~min}$ after glucose had been given. Results of the rising blood glucose level (Figure 2A) and the $\triangle \mathrm{AUC}$ (Figure $2 \mathrm{~B}$ ) in 25 and $50 \mathrm{mg} / \mathrm{kg}$ AFE-treated groups were significant as compared to untreated control. Additionally, hypoglycemic effect induced by AFE was observed in the highest dose used. However, $10 \mathrm{mg} / \mathrm{kg}$ AFE did not reduce blood glucose significantly and there was no hypoglycemic effect. In addition, there was statistical significance between lowest and highest dosages used $(p<0.001)$. The hypoglycemic effect induced by AFE was dose-dependent (Figures 2A,B) suggesting that AFE at doses of 25 and $50 \mathrm{mg} / \mathrm{kg}$ have potential to improve glucose intolerance of metabolic syndrome.

\section{AFE Does Not Change Blood Glucose in Non-hyperglycemic Mice}

The mice in the control group were intraperitoneally administrated with normal saline or 10,25 , and $50 \mathrm{mg} / \mathrm{kg}$ of AFE prior to glucose administration for glucose tolerance test. Neither raising blood glucose level (Figure 2C) nor $\triangle \mathrm{AUC}$ (Figure 2D) showed a significant difference during 2 h-study 


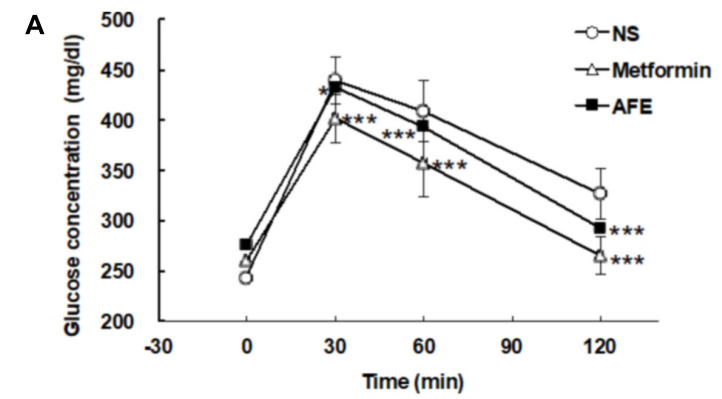

B

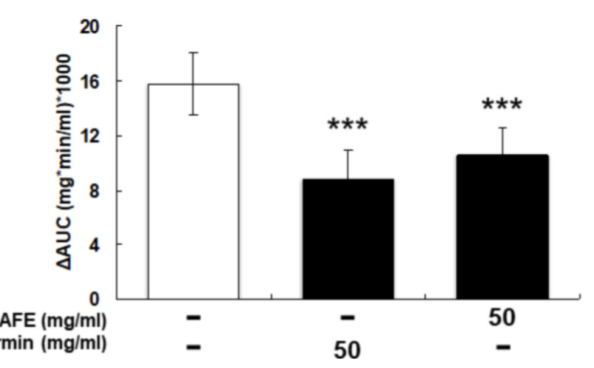

FIGURE 3 | Effect of AFE-reduced blood glucose is comparable to that of metformin in hyperglycemic mice. Hyperglycemic mice were injected with metformin $(50 \mathrm{mg} / \mathrm{kg}$ ) or AFE $(50 \mathrm{mg} / \mathrm{kg})$ intraperitoneally. Glucose was injected intraperitoneally after metformin or AFE administration. Blood glucose was measured at 0, 30, 60, and 120 min after glucose administration. (A) The time courses of blood glucose concentration measured in fructose-induced hyperglycemic mice treated with metformin and AFE $(50 \mathrm{mg} / \mathrm{kg})$. NS: treated with normal saline as control. Data are shown as mean $\pm S D(n=16)$. ${ }^{*} p<0.05,{ }^{* * *} p<0.001$, compared to untreated control group.

(B) Measurement of the area under the incremental blood glucose concentration curve in the glucose tolerance test of the experimental group mice. -: treated with normal saline as control; 50: treated with metformin or AFE $50 \mathrm{mg} / \mathrm{kg}$. Data are shown as mean \pm SD of each group $(n=16)$, analysis by one-way ANOVA test. ${ }^{* * *} p<0.001$ as compared with untreated control.

period. The results indicated that there was no significantly hypoglycemic effect induced by AFE at any concentration. The blood glucose dropped back to the baseline after $2 \mathrm{~h}$ indicating that the AFE would not affect blood glucose in control mice.

\section{AFE Has the Same Performance as Metformin to Reduce Blood Glucose in Hyperglycemic Mice}

The comparison between effects of AFE and metformin-induced hypoglycemic effect on blood glucose was conducted. After being fasted for $12 \mathrm{~h}$, tail vein blood was collected at 0 min from mice. A dose of $50 \mathrm{mg} / \mathrm{kg}$ AFE or metformin was injected intraperitoneally. Glucose solution $(1 \mathrm{~g} / \mathrm{kg})$ was given and mice were tested for blood glucose at 30, 60, and $120 \mathrm{~min}$ after glucose was given. The blood glucose concentrations were significant lower and similar after AFE and metformin administration as compared to those of control (Figure 3A). The $\triangle \mathrm{AUCs}$ induced by $50 \mathrm{mg} / \mathrm{kg}$ of metformin and AFE were also significant as compared to that of the control group (Figure 3B). Furthermore, the reducing effect of glucose by AFE $50 \mathrm{mg} / \mathrm{kg}$ was comparable to that of by metformin $50 \mathrm{mg} / \mathrm{kg}$. Although the $\triangle \mathrm{AUC}$ of AFE

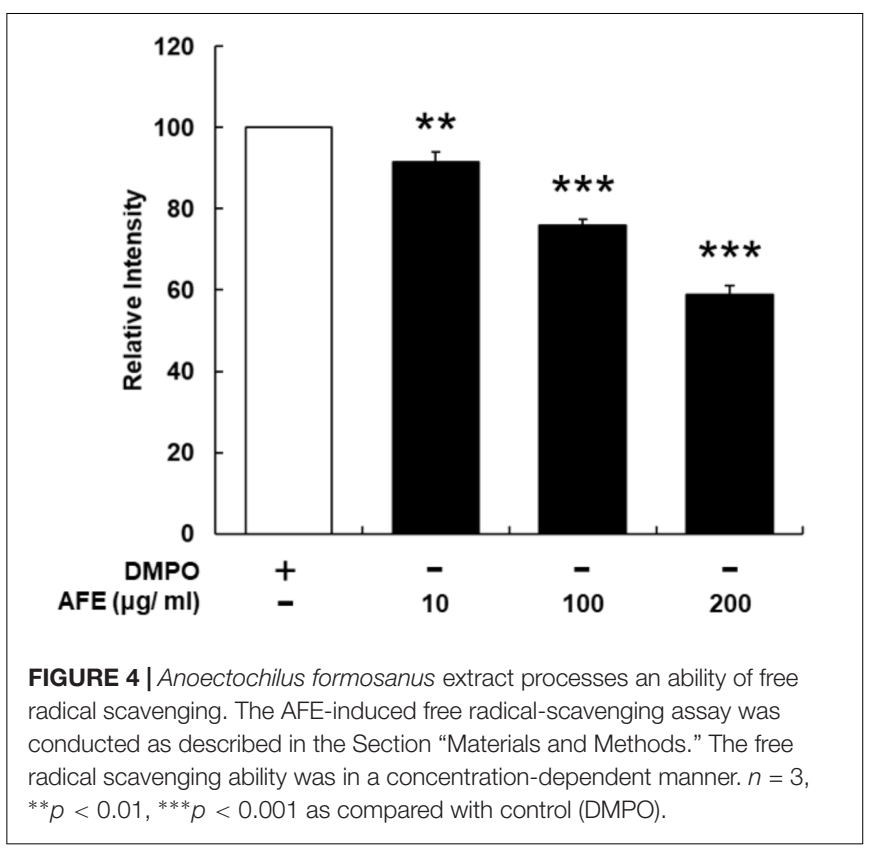

$50 \mathrm{mg} / \mathrm{kg}$ was greater than that of metformin administration group, there was no significant difference between those two drug-treated groups suggesting that the function of reducing blood glucose at AFE $50 \mathrm{mg} / \mathrm{kg}$ was similar or equivalent to that of metformin $50 \mathrm{mg} / \mathrm{kg}$.

\section{AFE Processes a Free Radical Scavenging Activity}

The hypoglycemic ability is related to the free radical scavenging ability and anti-proliferative effect on cancer cells, we next examined the free radical scavenging activity of AFE. Since AFE contains high total phenolic compounds and flavonoids 39.7 and $24.7 \mathrm{mg} / \mathrm{g}$ extract, respectively (data not shown), the free radical scavenging effect of AFE was predictable. Our ESR results indicated that AFE competed with DMPO for trapping free radicals starting at the concentration of $10 \mu \mathrm{g} / \mathrm{ml}$. The free radical scavenging ability was in a concentration-dependent manner. It removed more than $41.1 \pm 2.2 \%$ of free radicals at a concentration of $200 \mu \mathrm{g} / \mathrm{ml}$ (Figure 4).

\section{AFE Inhibits PD-L1 Expression in Oral Cancer Cells}

Recently, we have shown that resveratrol, a stilbenoid, inhibits constitutively expressed PD-L1 in cancer cells (Chin et al., 2018). Since AFE processes free radical scavenging activity, it was of interest to further investigate if AFE also inhibited the expression of $P D-L 1$ in cancer cells. Oral cancer SCC-25 cells were set in Petri dishes and starved for $48 \mathrm{~h}$ before being treated with AFE and metformin. Cells were treated with refreshed media with agents daily for 3 days. Total RNA was extracted and qPCR was conducted for the expression of $P D-L 1$. Parallel studies were conducted for Western blotting analysis of PD-L1 protein affected by AFE and metformin. Results indicated that AFE $(1 \mathrm{mg} / \mathrm{ml})$ shut down $P D-L 1$ expression completely (Figure 5A). 
A

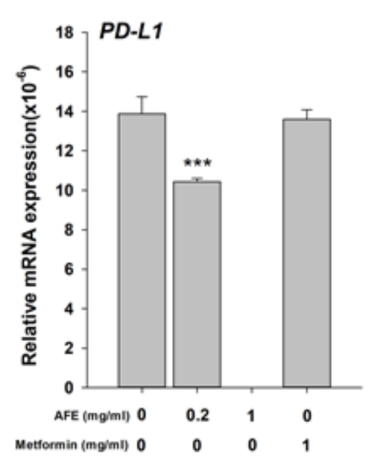

B

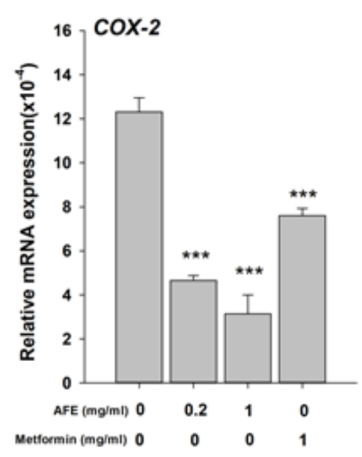

PD-L1

GAPDH
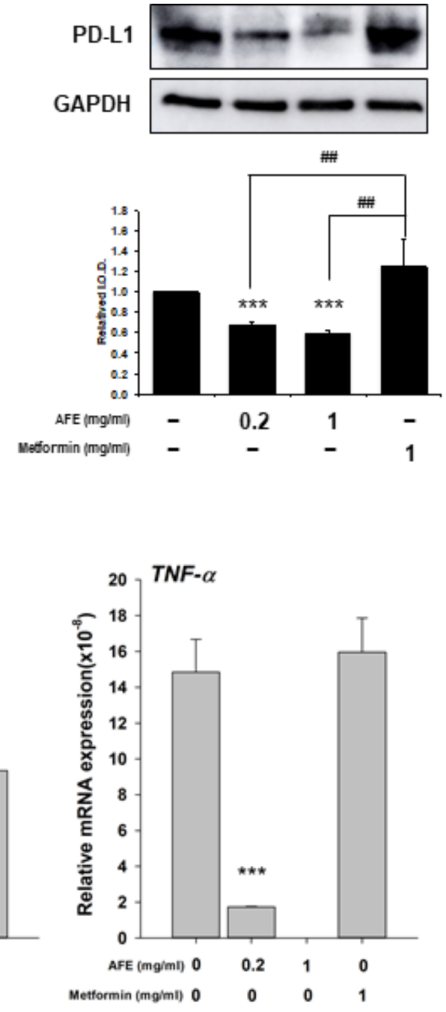
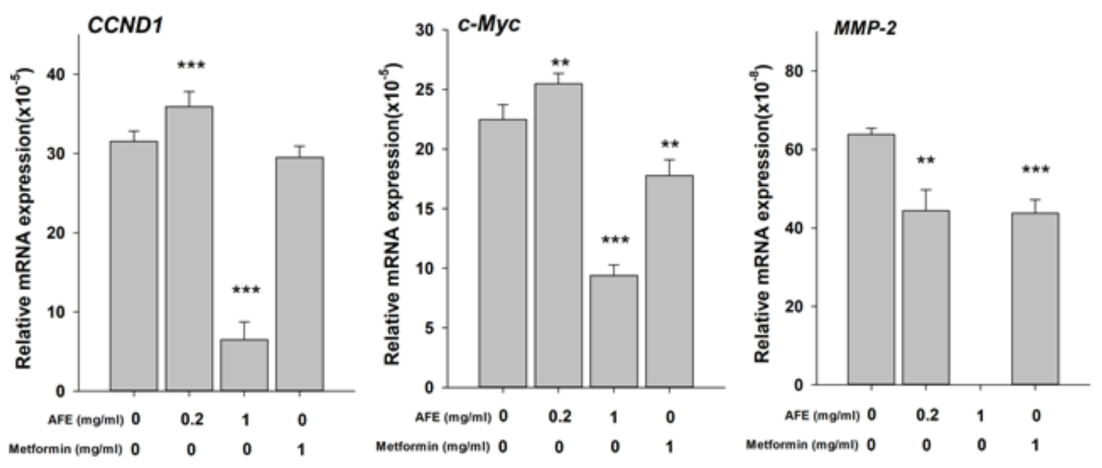

FIGURE 5 | Anoectochilus formosanus extract inhibits expression of PD-L1, inflammatory and proliferative genes in oral cancer SCC25 cells. SCC-25 cells were treated with different concentrations of AFE or metformin with refreshed media with agents daily for 3 days. Total RNA and protein were extracted. QPCR and Western blotting analysis were performed for (A) PD-L1 (left hand panel) and PD-L1 protein (right-hand pane/). (B) Parallel studies were conducted as described in (A). Total RNA was extracted and gPCR was performed for COX-2, TNF- $\alpha, C C N D-1, c-M y c$, and MMP-2. Numbers of independent studies $(n=3) * * p<0.01$, ${ }^{* * *} p<0.001$, as compared with control. ${ }^{\# \#} p<0.01$, as compared with metformin.

Meanwhile, same concentration of AFE $(1 \mathrm{mg} / \mathrm{ml})$ also inhibited PD-L1 protein accumulation (Figure 5A).

We further investigated the inhibitory effect of AFE and metformin on proliferative and anti-proliferative genes. AFE reduced inflammatory gene, $C O X-2$ and $T N F-\alpha$ (Figure 5B) and proliferative genes, Cyclin D1 (CCND1), $c-M y c$, and $M M P-2$, partially (Figure 5B). On the other hand, $1 \mathrm{mg} / \mathrm{ml}$ metformin did not inhibit expression of $P D-L 1$ and $T N F-\alpha$ (Figure 5B) and only inhibited $33 \%$ of $C O X-2$ expression at same treated concentration (Figure 5B).

Expression of two pro-apoptotic genes was examined. Expression of $B A D$ induced by $\operatorname{AFE}(0.2$ and $1 \mathrm{mg} / \mathrm{ml})$ was significantly but metformin $1 \mathrm{mg} / \mathrm{ml}$ inhibited $B A D$ expression (Figure 6A). Cell viability assay indicates that $1 \mathrm{mg} / \mathrm{ml}$ AFE inhibited oral cancer SCC-25 cell proliferation more than $82 \%$ (Figure 6B). However, treated with metformin (1 $\mathrm{mg} / \mathrm{ml}$ ) only inhibited $26 \%$ SCC25 cell growth which was similar to that of $0.2 \mathrm{mg} / \mathrm{ml} \mathrm{AFE} \mathrm{(Figure} \mathrm{6B).} \mathrm{On} \mathrm{the}$ other hand, expression of CASP2 by $0.2 \mathrm{mg} / \mathrm{ml}$ AFE was significant but $1 \mathrm{mg} / \mathrm{ml}$ AFE inhibited CASP2 expression (Figure 6A). There was no effect of metformin on CASP2 expression (Figure 6A). The results of free radical scavenging activity and inhibitory effect on $P D-L 1$ expression and cancer cell proliferation demonstrated that AFE may be used for 

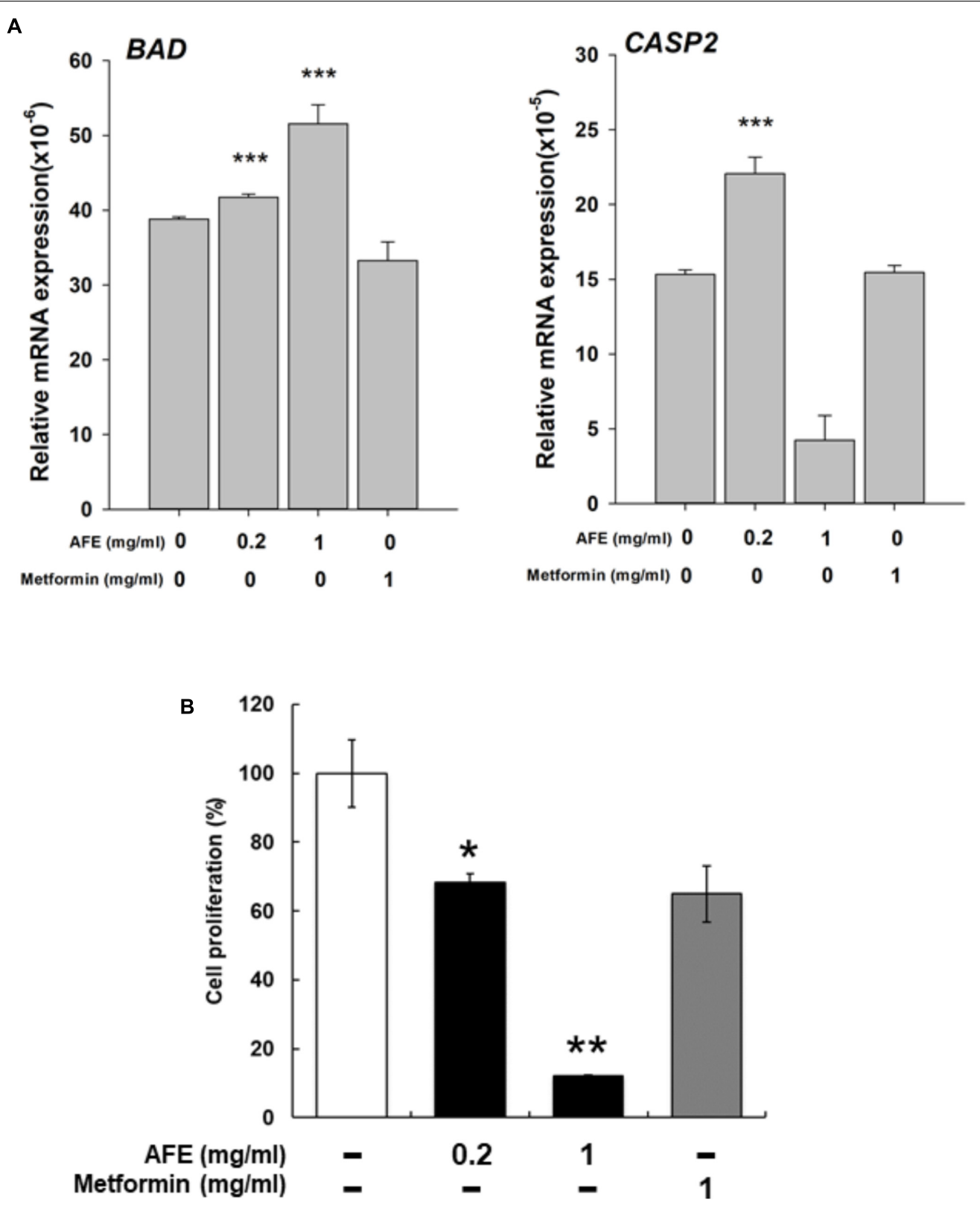

FIGURE 6 | Anoectochilus formosanus extract regulates expression of anti-proliferative genes and cell proliferation in oral cancer SCC25 cells. SCC-25 cells were treated with different concentrations of AFE or metformin with refreshed media with agents daily for 3 days. (A) Total RNA was extracted and qPCR was performed for BAD and CAPS2. $n=3^{* * *} p<0.001$ as compared with control. (B) SCC-25 cells were treated with AFE $(0.2$ and $1 \mathrm{mg} / \mathrm{ml})$ or metformin (1 mg/ml) with refreshed media with agents daily for 3 days. AFE induced anti-proliferation. -: treated with normal saline as control; $n=3 * p<0.05,{ }^{* *} p<0.01$ as compared with control.

cancer chemoprevention via suppressing $P D-L 1$ expression (Figure 5).

\section{DISCUSSION}

Metabolic abnormalities during the onset and development of diabetes may have a vital role on the initiation and progression of carcinogenesis. However, so far, no conclusive data are there on the mechanisms linked the relationship between diabetes and any type of human cancer. Nevertheless, recent evidence indicates that both hyperglycemia and hyperinsulinemia in diabetes may provoke cell damage responses, such as oxidative stress, glucotoxicity, and lipotoxicity involved in the cell transformation process to raise the risk of cancer development (Cignarelli et al., 2018). In addition, evidence has indicated that hyperglycemia promotes the acquirement of mesenchymal and cancer stem cells (CSC) properties in malignant pancreatic ductal epithelial cells by activating TGF- $\beta$ signaling (Rahn et al., 2018). 

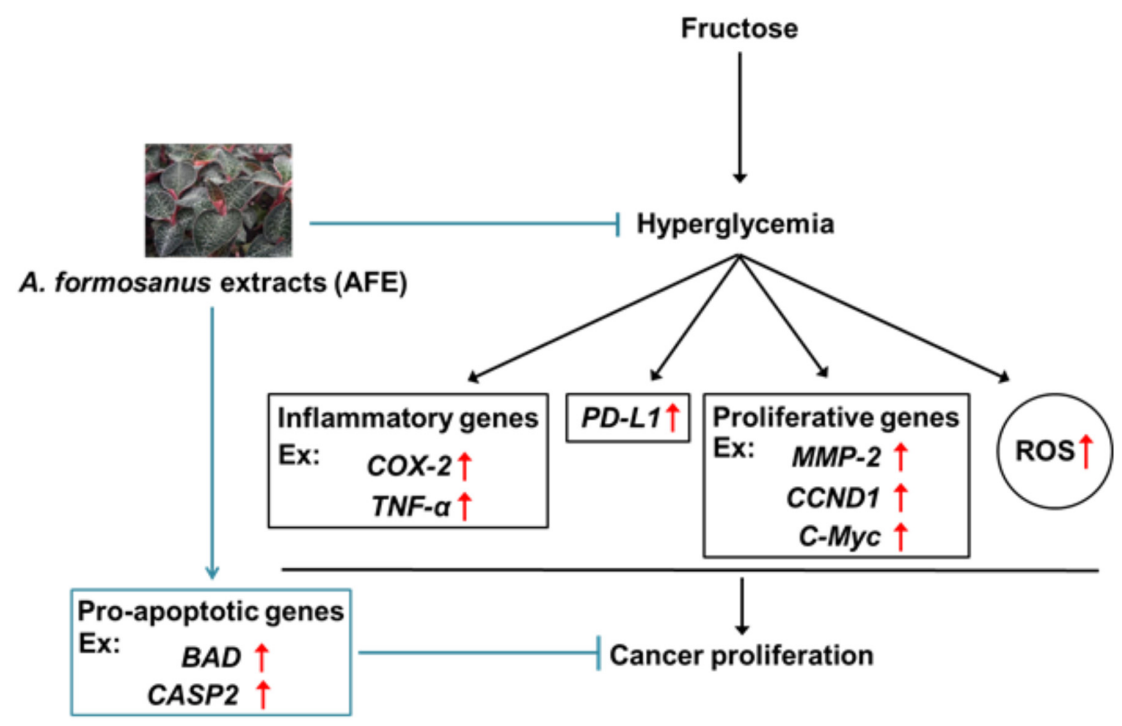

FIGURE 7 | Mechanism involved in Anoectochilus formosanus extract-induced anti-proliferation in cancer cells. Hyperglycemia modulates expression of inflammatory genes and increases ROS accumulation. A. formosanus extract can reduce ROS accumulation and inhibits PD- $L 1$ expression and further block expression of inflammatory, proliferative, and metastatic genes. All of them are important for anti-proliferation in cancer cells.

Results indicate that weight in $\mathrm{C} 57 \mathrm{BL} / 6$ mice with the fructose-induced hyperglycemic group were greater than those control group mice (Figure 1). Although mice in the experimental group had lower intake of diet than the control mice, most of the mice in the experimental group took fructose as caloric source, and experimental mice in the observation of activity were less active. Long-term consumption of excess fructose drink can cause hyperglycemia and obesity, which can easily lead to the occurrence of type II diabetes. In the fasting blood glucose results, there was a significant difference $(p<0.001)$ in fasting blood glucose level between the experimental group and the control group at the 11th week of induction (Figure 1).

Anoectochilus formosanus extract did not affect blood glucose level in untreated control mice (Figure 2) while the mice in the experimental group administered AFE, AFE could effectively improve glucose tolerance (Figures 2A,B) compared with untreated control mice. The blood glucose reduction is proportional to the AFE dose applied (Figure 3). This result is similar to that of fruit extracts of Eugenia uniflora or Psidium cattleianum which exhibit considerably anti-hyperglycemic, antidyslipidemic, and anti-oxidant effects, and may be useful in the therapeutic management of alterations due to insulin resistance (de Souza Cardoso et al., 2018). In addition, a naturally occurring polyphenolic stilbene, piceatannol has been reported to exhibit anticancer and anti-inflammatory properties and beneficial effects in hypercholesterolemia, atherosclerosis, and angiogenesis (Kershaw and Kim, 2017). Resveratrol has been reported to significantly decrease high glucose-induced production of ROS and $\mathrm{H}_{2} \mathrm{O}_{2}$ in Panc- 1 cells. It is also able to inhibit high glucoseinduced proliferation, migration, and invasion of pancreatic cancer cells companied with reducing expression of urokinase plasminogen activator (uPA) (Cao et al., 2016), E-cadherin, and Glut-1 modulated by high glucose. High glucose-induced activation of ERK and p38 MAPK signaling pathways as well as the transcription factor NF- $\kappa \mathrm{B}$ is suppressed by resveratrol (Cao et al., 2016).

Physiological regulation of blood glucose in the following mechanisms, such as the promotion of insulin secretion, inhibition of hepatic glycogenolysis and gluconeogenesis, slows down the decomposition of carbohydrates and promotes the surrounding tissue (such as skeletal muscle and fat cells) to uptake glucose, etc. Results were observed that AFE even had the effect of lowering blood glucose level in mice with hyperglycemia.

As hyperglycemia can be treated successfully and effectively with metformin, the complication by reducing the dose of or discontinuing the anticancer drug may be counterproductive, especially if it is otherwise effective and clinically tolerated. The use of metformin to treat hyperglycemia induced by anticancer drugs provides a valuable therapeutic opportunity of potentiating their clinical anticancer effects (Shah, 2017).

Results indicated that AFE particularly had similar hypoglycemic effects in the experimental mice group compared to those treated with $50 \mathrm{mg} / \mathrm{kg}$ metformin (Figure 4). Those observations in glucose tolerance test suggest that AFE has a significant function to reduce blood glucose which was equivalent to that of an oral dose of metformin. The latter has been used to reduce hyperglycemia induced by anti-cancer drugs (Shah, 2017). Indeed, metformin mimics caloric restriction acting on cell metabolism at multiple levels, reducing all energy-consuming processes in the cells, including cell proliferation (Pierotti et al., 2013). It greatly increases anti-tumor activity in mice with a functional immune system by increasing the number of CD8positive tumor-infiltrating lymphocytes in an AMPK-dependent manner (Eikawa et al., 2015). 
Results showed that AFE $(1 \mathrm{mg} / \mathrm{ml})$ inhibited constitute PDL1 expression completely and its protein accumulation partially (Figure 5A). AFE $(1 \mathrm{mg} / \mathrm{ml})$ also reduced inflammatory gene, $C O X-2$, proliferative genes, Cyclin D1 (CCND1), and $c-M y c$ and metastatic gene, $M M P-2$, partially (Figure $5 \mathbf{B}$ ). On the other hand, metformin did not inhibit $P D-L 1$ expression at the same concentration (Figure 5A). Cell viability assay indicates that $1 \mathrm{mg} / \mathrm{ml}$ AFE inhibited oral cancer SCC-25 cell proliferation by more than $82 \%$ (Figure 5B). However, only $26 \%$ was inhibited when treated with metformin $(1 \mathrm{mg} / \mathrm{ml})$. These results suggest that although both AFE and metformin can be used to prevent hyperglycemia, they may prevent cancer progression via different signal transduction pathways. Studies also suggest that AFE, via inhibiting hyperglycemia-related $P D-L 1$ expression, shuts down the expression of inflammatory and proliferative genes which play important roles in cancer proliferation and metastasis.

In summary, we provided evidence that via reducing blood glucose in hyperglycemia, AFE has free radical scavenging capability to inhibit $P D-L 1$ expression. Mechanism involved in AFE-induced anti-proliferation in cancer cells was via inhibition of $P D-L 1$ expression to inhibit expression of inflammatory, proliferative, and metastatic genes. In addition, it also stimulated apoptotic genes to further block cancer cell proliferation (Figure 7). Since AFE has similar hypoglycemic effect as metformin and the latter has been shown to reverse snit-cancer drug-induced hyperglycemia during cancer chemotherapy. We

\section{REFERENCES}

Agrawal, N. K., and Kant, S. (2014). Targeting inflammation in diabetes: newer therapeutic options. World J. Diabetes 5, 697-710. doi: 10.4239/wjd.v5.i5.697

Anisimov, V. N. (2016). Metformin for prevention and treatment of colon cancer: a reappraisal of experimental and clinical data. Curr. Drug Targets 17, 439-446. doi: 10.2174/1389450116666150309113305

Cade, W. T. (2008). Diabetes-related microvascular and macrovascular diseases in the physical therapy setting. Phys. Ther. 88, 1322-1335. doi: 10.2522/ptj. 20080008

Cao, L., Chen, X., Xiao, X., Ma, Q., and Li, W. (2016). Resveratrol inhibits hyperglycemia-driven ROS-induced invasion and migration of pancreatic cancer cells via suppression of the ERK and p38 MAPK signaling pathways. Int. J. Oncol. 49, 735-743. doi: 10.3892/ijo.2016.3559

Chin, Y. T., Wei, P. L., Ho, Y., Nana, A. W., Changou, C. A., Chen, Y. R., et al. (2018). Thyroxine inhibits resveratrol-caused apoptosis by PD-L1 in ovarian cancer cells. Endocr. Relat. Cancer 25, 533-545. doi: 10.1530/ERC-17-0376

Cignarelli, A., Gench, I. V. A., Caruso, I., Natalicchio, A., Perrini, S., Laviola, L., et al. (2018). Diabetes and cancer: pathophysiological fundamentals of a 'dangerous affair. Diabetes Res. Clin. Pract. doi: 10.1016/j.diabres.2018.04.002 [Epub ahead of print].

de Souza Cardoso, J., Oliveira, P. S., Bona, N. P., Vasconcellos, F. A., Baldissarelli, J., Vizzotto, M., et al. (2018). Antioxidant, antihyperglycemic, and antidyslipidemic effects of Brazilian-native fruit extracts in an animal model of insulin resistance. Redox Rep. 23, 41-46. doi: 10.1080/13510002.2017.1375709

Donadon, V., Balbi, M., Mas, M. D., Casarin, P., and Zanette, G. (2010). Metformin and reduced risk of hepatocellular carcinoma in diabetic patients with chronic liver disease. Liver Int. 30, 750-758. doi: 10.1111/j.1478-3231.2010.02223.x

Droeser, R. A., Hirt, C., Viehl, C. T., Frey, D. M., Nebiker, C., Huber, X., et al. (2013). Clinical impact of programmed cell death ligand 1 expression in colorectal cancer. Eur. J. Cancer 49, 2233-2242. doi: 10.1016/j.ejca.2013.02.015

Duan, W., Shen, X., Lei, J., Xu, Q., Yu, Y., Li, R., et al. (2014). Hyperglycemia, a neglected factor during cancer progression. Biomed Res. Int. 2014:461917. doi: $10.1155 / 2014 / 461917$ suggest that AFE would have beneficial effects for PD-L1 immunoprevention/therapy, singly or in combination with an anti-cancer drug, particularly in patients with tumors that express $P D-L 1$ and are under hyperglycemic condition.

\section{AUTHOR CONTRIBUTIONS}

YH designed the experiments, performed the experiments, and analyzed the data. KW designed the experiments. L-HW and K-YH analyzed the results. Y-FC, Y-TC, Y-CY, S-HW, Y-RC, and Y-JS performed the experiments and collected the data. LL, KW, JW-P, H-YT, H-YL, H-LL, and S-JL in wrote the manuscript and supervised the work.

\section{FUNDING}

This work was supported in part by collaborating grants of TMU and National Taipei University of Technology (NTUT-TMU103-06 and USTP-NTUT-TMU-104-09), the "TMU Research Center of Cancer Translational Medicine" from The Featured Areas Research Center Program within the framework of the Higher Education Sprout Project by the Ministry of Education (MOE) in Taiwan and grants from Ministry of Science and Technology, Taiwan (MOST 104-2314-B-038-046MY3; MOST105-2320-B-038-006).

Eikawa, S., Nishida, M., Mizukami, S., Yamazaki, C., Nakayama, E., and Udono, H. (2015). Immune-mediated antitumor effect by type 2 diabetes drug, metformin. Proc. Natl. Acad. Sci. U.S.A. 112, 1809-1814. doi: 10.1073/pnas.14176 36112

Fainsod-Levi, T., Gershkovitz, M., Vols, S., Kumar, S., Khawaled, S., Sagiv, J. Y., et al. (2017). Hyperglycemia impairs neutrophil mobilization leading to enhanced metastatic seeding. Cell Rep. 21, 2384-2392. doi: 10.1016/j.celrep. 2017.11.010

Goncalves, A. S., Mosconi, C., Jaeger, F., Wastowski, I. J., Aguiar, M. C. F., Silva, T. A., et al. (2017). Overexpression of immunomodulatory mediators in oral precancerous lesions. Hum. Immunol. 78, 752-757. doi: 10.1016/j.humimm. 2017.09.003

Gothai, S., Ganesan, P., Park, S.-Y., Fakurazi, S., Choi, D.-K., and Arulselvan, P. (2016). Natural phyto-bioactive compounds for the treatment of type 2 Diabetes: inflammation as a target. Nutrients 8:E461. doi: 10.3390/nu8080461

Kasznicki, J., Sliwinska, A., and Drzewoski, J. (2014). Metformin in cancer prevention and therapy. Ann. Transl. Med. 2:57.

Kershaw, J., and Kim, K. H. (2017). The therapeutic potential of piceatannol, a natural stilbene, in metabolic diseases: a review. J. Med. Food 20, 427-438. doi: 10.1089/jmf.2017.3916

Kitabchi, A. E., Umpierrez, G. E., Miles, J. M., and Fisher, J. N. (2009). Hyperglycemic crises in adult patients with diabetes. Diabetes Care 32, 1335-1343. doi: 10.2337/dc09-9032

Li, W., Cao, L., Han, L., Xu, Q., and Ma, Q. (2015). Superoxide dismutase promotes the epithelial-mesenchymal transition of pancreatic cancer cells via activation of the H2O2/ERK/NF-kappaB axis. Int. J. Oncol. 46, 2613-2620. doi: 10.3892/ijo. 2015.2938

Li, W., Ma, Q., Li, J., Guo, K., Liu, H., Han, L., et al. (2011). Hyperglycemia enhances the invasive and migratory activity of pancreatic cancer cells via hydrogen peroxide. Oncol. Rep. 25, 1279-1287. doi: 10.3892/or.2011.1150

Li, W., Zhang, L., Chen, X., Jiang, Z., Zong, L., and Ma, Q. (2016). Hyperglycemia promotes the epithelial-mesenchymal transition of pancreatic cancer via hydrogen peroxide. Oxid. Med. Cell. Longev. 2016:5190314. doi: 10.1155/2016/ 5190314 
Lim, S. O., Li, C. W., Xia, W., Cha, J. H., Chan, L. C., Wu, Y., et al. (2016). Deubiquitination and stabilization of PD-L1 by CSN5. Cancer Cell 30, 925-939. doi: 10.1016/j.ccell.2016.10.010

Lin, H. Y., Chin, Y. T., Nana, A. W., Shih, Y. J., Lai, H. Y., Tang, H. Y., et al. (2016). Actions of l-thyroxine and Nano-diamino-tetrac (Nanotetrac) on PD-L1 in cancer cells. Steroids 114, 59-67. doi: 10.1016/j.steroids.2016. 05.006

Navarro, J. F., and Mora, C. (2005). Role of inflammation in diabetic complications. Nephrol. Dial. Transplant. 20, 2601-2604. doi: 10.1093/ndt/gfi155

Nishikawa, M., Hashida, M., and Takakura, Y. (2009). Catalase delivery for inhibiting ROS-mediated tissue injury and tumor metastasis. Adv. Drug Deliv. Rev. 61, 319-326. doi: 10.1016/j.addr.2009.01.001

Pierotti, M. A., Berrino, F., Gariboldi, M., Melani, C., Mogavero, A., Negri, T., et al. (2013). Targeting metabolism for cancer treatment and prevention: metformin, an old drug with multi-faceted effects. Oncogene 32, 1475-1487. doi: 10.1038/ onc. 2012.181

Rahn, S., Zimmermann, V., Viol, F., Knaack, H., Stemmer, K., Peters, L., et al. (2018). Diabetes as risk factor for pancreatic cancer: hyperglycemia promotes epithelial-mesenchymal-transition and stem cell properties in pancreatic ductal epithelial cells. Cancer Lett. 415, 129-150. doi: 10.1016/j.canlet.2017.12.004

Ryu, T. Y., Park, J., and Scherer, P. E. (2014). Hyperglycemia as a risk factor for cancer progression. Diabetes Metab. J. 38, 330-336. doi: 10.4093/dmj.2014. 38.5.330

Shah, R. R. (2017). Hyperglycaemia induced by novel anticancer agents: an undesirable complication or a potential therapeutic opportunity? Drug Saf. 40, 211-228. doi: 10.1007/s40264-016-0485-y

Shih, C. C., Wu, Y. W., and Lin, W. C. (2002). Antihyperglycaemic and anti-oxidant properties of Anoectochilus formosanus in diabetic rats. Clin. Exp. Pharmacol. Physiol. 29, 684-688. doi: 10.1046/j.1440-1681.2002.03717.x

Shih, C. C., Wu, Y. W., and Lin, W. C. (2003). Scavenging of reactive oxygen species and inhibition of the oxidation of low density lipoprotein by the aqueous extraction of Anoectochilus formosanus. Am. J. Chin. Med. 31, 25-36. doi: 10.1142/S0192415X03000692

Simon, J. M., Thomas, F., Czernichow, S., Hanon, O., Lemogne, C., Simon, T., et al. (2018). Hyperglycaemia is associated with cancer-related but not non-cancerrelated deaths: evidence from the IPC cohort. Diabetologia 61, 1089-1097. doi: 10.1007/s00125-017-4540-8

Thierauf, J., Veit, J. A., Affolter, A., Bergmann, C., Grunow, J., Laban, S., et al. (2015). Identification and clinical relevance of PD-L1 expression in primary mucosal malignant melanoma of the head and neck. Melanoma Res. 25, 503-509. doi: 10.1097/CMR.0000000000000197

Trachootham, D., Alexandre, J., and Huang, P. (2009). Targeting cancer cells by ROS-mediated mechanisms: a radical therapeutic approach? Nat. Rev. Drug Discov. 8, 579-591. doi: 10.1038/nrd2803

Wu, W. S., Wu, J. R., and Hu, C. T. (2008). Signal cross talks for sustained MAPK activation and cell migration: the potential role of reactive oxygen species. Cancer Metastasis Rev. 27, 303-314. doi: 10.1007/s10555-008-9112-4

Zhuan-Sun, Y., Huang, F., Feng, M., Zhao, X., Chen, W., Zhu, Z., et al. (2017). Prognostic value of PD-L1 overexpression for pancreatic cancer: evidence from a meta-analysis. Onco Targets Ther. 10, 5005-5012. doi: 10.2147/OTT.S146383

Conflict of Interest Statement: The authors declare that the research was conducted in the absence of any commercial or financial relationships that could be construed as a potential conflict of interest.

Copyright (c) 2018 Ho, Chen, Wang, Hsu, Chin, Yang, Wang, Chen, Shih, Liu, Wang, Whang-Peng, Tang, Lin, Liu and Lin. This is an open-access article distributed under the terms of the Creative Commons Attribution License (CC BY). The use, distribution or reproduction in other forums is permitted, provided the original author(s) and the copyright owner(s) are credited and that the original publication in this journal is cited, in accordance with accepted academic practice. No use, distribution or reproduction is permitted which does not comply with these terms. 